\title{
Robust Maximum Weighted Independent-Set Problems on Interval Graphs
}

\author{
Fabrice Talla Nobibon • Roel Leus
}

Received: date / Accepted: date

\begin{abstract}
We study the maximum weighted independent-set problem on interval graphs with uncertainty on the vertex weights. We use the absolute robustness criterion and the min-max regret criterion to evaluate solutions. For a discrete scenario set, we find that the problem is NP-hard for each of the robustness criteria; we also provide pseudo-polynomial time algorithms when there is a constant number of scenarios and show that the problem is strongly NP-hard when the set of scenarios is unbounded. When the scenario set is a Cartesian product, we prove that the problem is equivalent to a maximum weighted independent-set problem on the same interval graph but without uncertainty for the first objective function and that the scenario set can be reduced for the second objective function.
\end{abstract}

Keywords combinatorial problems · computational complexity · interval graphs $\cdot$ independent set $\cdot$ dynamic programming

\section{Introduction}

Many real-life problems are modeled using interval graphs $[6,9,11,14]$. The solution procedure of some of these problems involves solving a maximum weighted independent-set problem (MWIS) where a weight is assigned to each vertex of the interval graph $[8,11,14]$. An important advantage of these models is the fact that they can be solved in polynomial time $[5,11,12,14]$. In practice,

Fabrice Talla Nobibon $(\bowtie)$

PostDoc researcher for Research Foundation - Flanders

QuantOM, HEC-Management School, University of Liège, Belgium

ORSTAT, Faculty of Business and Economics, KU Leuven, Belgium Tel.: +32 16326960

E-mail: Fabrice.TallaNobibon@econ.kuleuven.be

Roel Leus

ORSTAT, Faculty of Business and Economics, KU Leuven, Belgium 
however, the weights of the vertices are often known only approximatively and can take different values $[2,14]$. These imprecisions are mainly due to the lack of full information about the parameters of the problem and/or the dependence of these parameters on some uncontrolled events $[10,14]$. The outputs of the deterministic models (where the weight of each vertex is fixed to a single value) usually suffer from these imprecisions, which can make their practical implementation almost impossible in some cases [10]. Recently, researchers have started to develop methods to cope with such uncertainty. Two situations can be distinguished: either the decision maker is able to determine the appropriate probability distributions for modeling the uncertain elements and then builds and solves stochastic models [13], or there is no clear characterization of the uncertainty and all possible scenarios affecting the parameters of the problem are considered $[1,3,10]$. The latter setting is studied in this article.

The direct motivation for the work undertaken is the article by Saha et al. [14], who investigate the selection of program slots of television channels for running an advertisement such as to maximize the number of viewers of the selected programs. More concretely, the authors associate a vertex with each TV program slot and create an edge between two vertices if and only if the corresponding program timeslots have a non-empty intersection. Furthermore, a weight is assigned to each vertex that reflects the number of viewers of that program. This weight is not fixed but can take any value within a specific interval. The goal is to select the appropriate programs across different channels in order to reach the maximum number of viewers with the constraint that the selected programs do not overlap in time. Saha et al. [14] model the weight of each vertex as an interval number and solve the corresponding MWIS problem on an interval graph. Their analysis assumes that the number of viewers of a given TV program is independent of the numbers for the other programs. Concurrent TV programs naturally share the total viewer population, however, and with this possible dependence among the weights of the vertices, the approach pursued by Saha et al. is no longer valid.

The goal of the current paper is to propose a general framework that includes both the independent setting, where each weight can take any value within the given interval, as well as the dependent setting, where the possible weight combinations are limited. For lack of stochastic information, we tackle the problem from a worst-case viewpoint, optimizing the worst-case performance of the proposed solution. Concretely, we investigate robust solution procedures for MWIS on interval graphs, meaning that the produced solutions are, up to a certain point, immune to data uncertainty [4]. In the rest of this paper, we refer to these problems as robust maximum weighted independent-set problems (RMWIS). The uncertainties affecting the weights of the vertices are described by a discrete set of scenarios, and we also look into the special case where this set is a Cartesian product. To evaluate the quality of a solution, we use the absolute robustness criterion and the min-max regret criterion. For more detail about these criteria and their practical interpretation, we refer to Kouvelis and $\mathrm{Yu}[10]$. 
The remainder of this paper is organized as follows. First, we provide a formal description of the problems to be studied in Section 2, Section 3 contains our results for RMWIS with absolute robustness criterion and Section 4 is devoted to RMWIS with the min-max regret criterion. We conclude in Section 5 .

\section{Definitions and problem statement}

We write an interval as $I_{i}=\left[a_{i}, b_{i}\right]$, where $a_{i}, b_{i} \in \mathbb{R}$ and $a_{i}<b_{i}$. An undirected graph $G=(V, E)$ with $|V|=n$ vertices and $|E|=m$ edges is called an interval graph for a finite family $\mathcal{I}=\left\{I_{1}, I_{2}, \ldots, I_{n}\right\}$ of $n$ non-empty intervals on the real line if there is a one-to-one correspondence between $\mathcal{I}$ and $V$ such that two intervals in $\mathcal{I}$ have non-empty intersection if and only if their corresponding vertices in $V$ are adjacent to each other. In the rest of this paper, we assume that the set $\mathcal{I}$ contains closed intervals and is such that no two intervals share a common endpoint. We also assume that the intervals are sorted in increasing order of their right endpoint $b_{i}$ and that the interval $I_{i} \in \mathcal{I}$ corresponds to vertex $i$ in $V(i=1, \ldots, n)$. An independent set of $G$ is a subset of vertices of $G$ not containing two adjacent vertices.

An interval graph $G=(V, E)$ is weighted if each vertex $i \in V$ is associated with a number $w_{i}$ (the weight of $i$ ). In this note, we consider the weights $w_{i}$ to be non-negative integers. A non-empty discrete set $S$ contains the scenarios: each $s \in S$ represents an $n$-vector $W^{s}=\left(w_{1}^{s}, \ldots, w_{n}^{s}\right)$ where $w_{i}^{s}$ is the weight of vertex $i$ under scenario $s$. For a given interval graph, each $s \in S$ corresponds to an MWIS instance defined by:

$$
\begin{array}{rll}
\left(\mathrm{MWIS}_{s}\right) \quad \max _{X} & F_{s}(X)=\sum_{i=1}^{n} w_{i}^{s} x_{i} & \\
\text { s.t. } & x_{i}+x_{j} \leq 1 & \\
& x_{i} \in\{0,1\} & i=1, \ldots, n .
\end{array}
$$

In this notation, vector $X=\left(x_{1}, \ldots, x_{n}\right)$. Let $F_{s}^{*}$ be the optimal value of MWIS $_{s}$. Let $K$ be the set of all independent sets of $G$, i.e., $K=\left\{X \in\{0,1\}^{n}\right.$ : $\left.x_{i}+x_{j} \leq 1, \forall(i, j) \in E\right\}$; where $x_{i}=1$ if and only if vertex $i$ is in the independent set. For a given solution $X \in K$, the regret of $X$ under the scenario $s$ is the value $F_{s}^{*}-F_{s}(X)$ and the maximum regret $Z(X)$ of $X$ is defined by: $Z(X)=\max \left\{F_{s}^{*}-F_{s}(X): s \in S\right\}$. In this paper, we examine RMWIS with two objective functions, which will be the topics of Section 3 and Section 4.

\section{Absolute robustness}

In this section, we study RMWIS with the absolute robustness criterion, given by:

$$
\text { (AbRMWIS) } \quad \max _{X \in K} \min _{s \in S} F_{s}(X) \text {. }
$$


Below, we show that this problem is NP-hard. In the proof, we refer to the even-odd partition problem (EOPP), which is defined as follows:

Instance: A finite set $A=\{1,2, \ldots, 2 n\}$ with size $a_{i} \in \mathbb{Z}^{+} \backslash\{0\}$ for each $i \in A$, and $Q=\frac{1}{2} \sum_{i \in A} a_{i}$.

Question: Does there exist a subset $A^{\prime} \subset A$ with $\left|A^{\prime}\right|=n, \sum_{i \in A^{\prime}} a_{i}=$ $Q$ and precisely one of $2 i, 2 i-1$ belongs to $A^{\prime}$, for $i=1, \ldots, n$ ?

EOPP is NP-hard [7].

Proposition 3.1 AbRMWIS is NP-hard even when $S$ contains only two scenarios.

Proof We describe a reduction from EOPP. Given an arbitrary instance of EOPP, we build an interval graph $G=(V, E)$ with $4 n-2$ vertices. The set $E$ is built as follows. Given a vertex $i$,

- if $i=2 \ell-1$ with $1 \leq \ell \leq 2 n-1$, then the edges $\{i, i+1\}$ and $\{i, i+2\}$ are in $E$ provided that $i+2 \leq 4 n-2$, and the edge $\{i, i+3\} \in E$ when $i+3 \leq 4 n-2$.

- if $i=2 \ell$ with $1 \leq \ell \leq 2 n-1$, then edge $\{i, i+1\} \in E$ provided that $i+1 \leq 4 n-2$, and $\{i, i+2\} \in E$ on condition that $i+2 \leq 4 n-2$.

It can be verified that $G$ is an interval graph. To complete the description of the AbRMWIS instance, we next describe the set $S$ with two scenarios:

$$
\begin{aligned}
& w_{i}^{1}= \begin{cases}a_{2 \ell+1} & \text { if } i=4 \ell+1, \quad \ell=0,1, \ldots, n-1, \\
a_{2 \ell+2} & \text { if } i=4 \ell+2, \quad \ell=0,1, \ldots, n-1, \\
0 & \text { otherwise, }\end{cases} \\
& w_{i}^{2}= \begin{cases}a_{2 \ell+2} & \text { if } i=4 \ell+1, \quad \ell=0,1, \ldots, n-1, \\
a_{2 \ell+1} & \text { if } i=4 \ell+2, \quad \ell=0,1, \ldots, n-1, \\
0 & \text { otherwise. }\end{cases}
\end{aligned}
$$

We now show that the EOPP instance is a YES instance if and only if the optimal value of the instance of AbRMWIS is greater than or equal to $Q$.

On the one hand, suppose that the answer to the EOPP instance is YES, so an appropriate subset $A^{\prime}$ exists. Consider the set of vertices $\mathcal{C}=\{4 \ell+1$ : $\left.2 \ell+1 \in A^{\prime}, \ell=0,1, \ldots, n-1\right\} \cup\left\{4 \ell+2: 2 \ell+2 \in A^{\prime}, \ell=0,1, \ldots, n-1\right\}$; this is an independent set in $G(V, E)$ with exactly $n$ vertices. Additionally, the solution $X \in\{0,1\}^{4 n-2}$ corresponding with $\mathcal{C}$ is such that the total weight for any scenario is exactly $Q$.

On the other hand, suppose that the instance of AbRMWIS has optimal objective value greater than or equal to $Q$ (note that $Q$ is also an upper bound on the optimal value). Let $\mathcal{C}$ be an optimal solution (independent set); observe that it is a subset of $\{4 \ell+1,4 \ell+2: \ell=0,1, \ldots, n-1\}$ since these are the only vertices with positive weights. We construct the set $A^{\prime}=\{2 \ell+1$ : $4 \ell+1 \in \mathcal{C}, \ell=0,1, \ldots, n-1\} \cup\{2 \ell+2: 4 \ell+2 \in \mathcal{C}, \ell=0,1, \ldots, n-1\}$, which can be seen to fulfill all the conditions required for the OEPP instance to have a YES answer. 
We also obtain the following positive result:

Proposition 3.2 AbRMWIS can be solved in pseudo-polynomial time when $S$ is bounded.

Below, we provide a pseudo-polynomial-time algorithm for solving AbRMWIS when $S$ is bounded by a constant. To derive this algorithm, we first adapt the instance of AbRMWIS by adding two new vertices, namely vertex 0 and vertex $n+1$, each with weight 0 under any scenario. These two vertices are neither adjacent to each other nor to any other vertex in $V$. For ease of exposition, the adapted graph is also called $G$. We recall that the vertices of $G$ are ordered such that for two vertices $i$ and $j$ with $i<j$, the corresponding intervals $I_{i}=\left[a_{i}, b_{i}\right]$ and $I_{j}=\left[a_{j}, b_{j}\right]$ satisfy $b_{i}<b_{j}$. Our algorithm is based on dynamic programming (DP). We use the following value function:

$F_{k}\left(\alpha_{1}, \ldots, \alpha_{|S|}\right) \equiv$ the maximum of the minimum weight over all scenarios for an independent set included in $\{k+1, \ldots, n+1\}$ without vertex adjacent to $k$, if weight $\alpha_{s}$ is added to the objective under scenario $s$.

The quantity $\alpha_{s}$ represents the weights collected for scenario $s \in S$ when selecting an independent set included in $\{0,1, \ldots, k\}$ and containing the vertex $k$. An initial condition is specified by: $F_{n+1}\left(\alpha_{1}, \ldots, \alpha_{|S|}\right)=\min _{s \in S} \alpha_{s}$. A recursive relation for the remaining vertices is formulated as follows:

$$
F_{k}\left(\alpha_{1}, \ldots, \alpha_{|S|}\right)=\max _{k<\ell \leq n+1, I_{k} \cap I_{\ell}=\emptyset}\left\{F_{\ell}\left(\alpha_{1}+w_{\ell}^{1}, \ldots, \alpha_{|S|}+w_{\ell}^{|S|}\right)\right\} .
$$

The optimal value to AbRMWIS is $F_{0}(0, \ldots, 0)$.

The role of the value function $F_{k}$ can be understood as follows: for a given vertex $k$, define $\sigma_{k}$ as the set of independent sets from $\{k+1, \ldots, n+1\}$ that do not contain any vertex adjacent to vertex $k$. It can be seen that

$$
F_{k}\left(\alpha_{1}, \ldots, \alpha_{|S|}\right)=\max _{\beta \in \sigma_{k}} \min _{s \in S}\left\{\alpha_{s}+\sum_{\ell \in \beta} w_{\ell}\right\} .
$$

In other words, apart from the terms $\alpha_{s}$, this is the objective value of a smaller AbRMWIS instance. The recursive relation chooses among the vertices $k+1, \ldots, n+1$ an appropriate vertex to be added to the best independent set already built from vertices $0,1, \ldots, k$ and containing $k$; the condition of non-adjacency to $k$ ensures that the resulting "partial" independent set can be merged with one obtained from vertices $0,1, \ldots, k$ and that does contain vertex $k$ (and which adds values $\alpha_{s}$ to the weights of the scenarios). The initial condition follows from the fact that $\sigma_{n+1}=\{\emptyset\}$, with a unique (empty) independent set and hence only the minimization remains in (3.1). Finally, the optimal objective value is $F_{0}(0, \ldots, 0)$ because 0 is the first vertex and therefore no weight from "earlier" partial independent sets needs to be included. Put differently, $\sigma_{0}$ contains all the feasible solutions to the initial instance to be solved. 


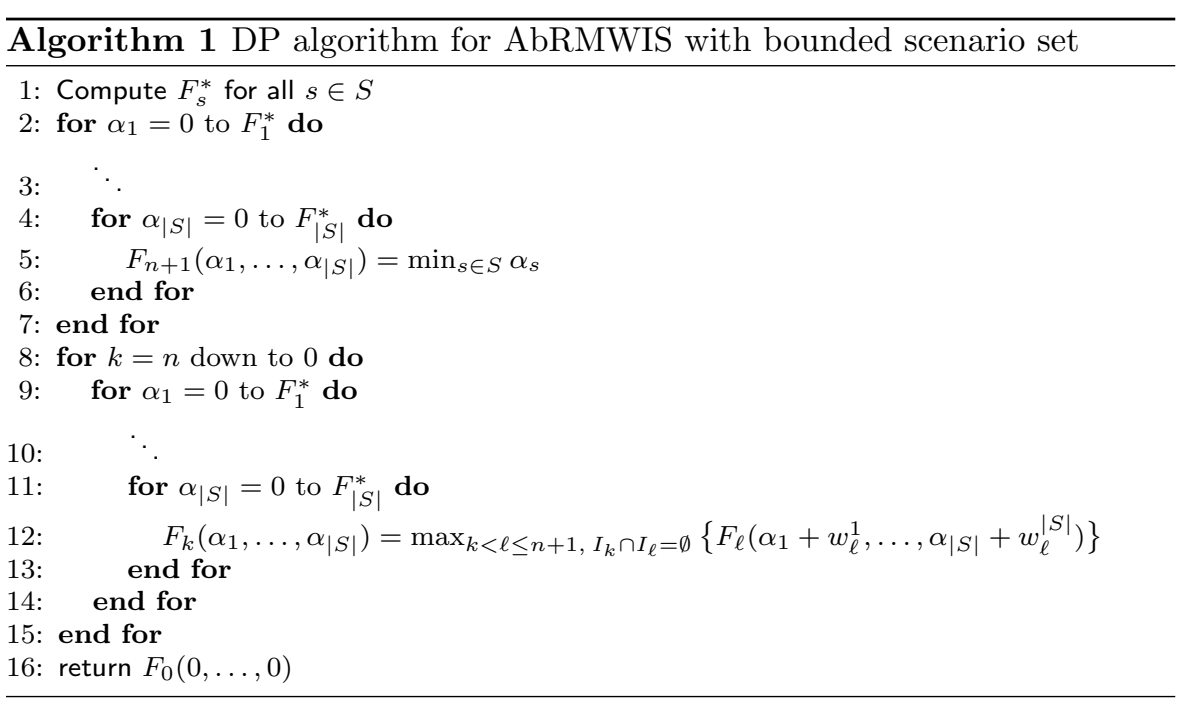

The complete pseudocode of the procedure is given by Algorithm 1, and the time complexity is $O\left(|S| n \log n+n L^{|S|}\right)$, where $L=\max _{s \in S} F_{s}^{*}$ and each value $F_{s}^{*}$ can be computed in time $O(n \log n)$ [11]. Thus, if $|S|$ is bounded by a constant, the algorithm runs in pseudo-polynomial time.

We define the problem 3-partition, which is strongly NP-hard [7]:

Instance: A finite set $A=\{1,2, \ldots, 3 m\}$, a bound $B \in \mathbb{Z}^{+}$, and a size $a_{i} \in \mathbb{Z}^{+}$for each $i \in A$ such that $\frac{B}{4}<a_{i}<\frac{B}{2}$ and such that $\sum_{i \in A} a_{i}=m B$.

Question: Can $A$ be partitioned into $m$ disjoint sets $A_{1}, A_{2}, \ldots, A_{m}$ such that for $1 \leq k \leq m, \sum_{i \in A_{k}} a_{i}=B$ ?

Proposition 3.3 AbRMWIS is strongly NP-hard when $S$ is unbounded.

Proof The proof uses a reduction from 3-partition. Given an arbitrary instance of the 3-partition problem, we build an interval graph $G=(V, E)$ as the union of $3 m$ disjoint interval graphs $G_{k}=\left(V_{k}, E_{k}\right)$ for $1 \leq k \leq 3 m$. Each graph $G_{k}$ is a complete graph with $m$ vertices $\left\{v_{k i} \mid i=1, \ldots, m\right\}$. The reader can easily verify that $G$ is an interval graph. The set $S$ contains $m$ scenarios defined as follows:

$$
w_{v_{k i}}^{s}=\left\{\begin{array}{l}
a_{k} \text { if } i=s \\
0 \text { otherwise. }
\end{array}\right.
$$

We claim that there exists a 3-partition if and only if the instance of AbRMWIS built above has an optimal value of $B$.

On the one hand, suppose there exists a 3 -partition of $A$ into $A_{1}, A_{2}, \ldots$, $A_{m}$ with $\sum_{i \in A_{k}} a_{i}=B, k=1, \ldots, m$. An optimal solution to the instance of AbRMWIS can be found by considering the independent set $\mathcal{C}$ containing the vertices $v_{i k}$ for $i \in A_{k}, k=1, \ldots, m$. This is an independent set with a total weight of $B$ for each scenario. 
On the other hand, suppose that the instance of AbRMWIS has an indepen$\operatorname{dent}$ set $\mathcal{C}$ with optimal value $B$. For each $k=1, \ldots, m$, let $A_{k}=\left\{i \mid v_{i k} \in \mathcal{C}\right\}$. By the structure of $G$, it is clear that the sets $A_{k}$ are disjoint. It also holds that the total weight of $\mathcal{C}$ under scenario $k$ is $\sum_{i \in A_{k}} w_{v_{i k}}^{k}=\sum_{i \in A_{k}} a_{i}(k \in$ $\{1, \ldots, m\})$. Since by definition the optimal value of AbRMWIS is $B$, we have $\sum_{i \in A_{k}} a_{i}=B$ for all $A_{k}$, and so the set of sets $A_{k}$ constitutes a 3 -partition.

We now examine the special case where $S$ is a Cartesian product. Suppose that the weight $w_{i}$ of vertex $i$ can take any value in a given set $W_{i}$ and let the scenario set be the Cartesian product of the $W_{i}$, so $S=\prod_{i=1}^{n} W_{i}$. This corresponds to the situation where the weight of one vertex does not depend on the particular weights of the other vertices. Let $L_{i}=\min \left\{w \in W_{i}\right\}$ and consider the scenario $\bar{s} \in S$ defined by $w_{i}^{\bar{s}}=L_{i}$ for each vertex $i$ and let MWIS $_{\bar{s}}$ be the associated MWIS problem. The next result shows that in the case of Cartesian scenarios, a solution to AbRMWIS is obtained by solving $\mathrm{MWIS}_{\bar{s}}$.

Proposition 3.4 An optimal solution to AbRMWIS is obtained by solving $M W I S_{\bar{s}}$.

Proof Let $\Delta$ be the optimal value of AbRMWIS and $\Delta_{\bar{s}}$ be that of MWIS $_{\bar{s}}$; we want to show that $\Delta=\Delta_{\bar{s}}$. On the one hand, since $\bar{s} \in S$ we have $\Delta \leq$ $\Delta_{\bar{s}}$. On the other hand, let $X \in K$ be an independent set. For each $s \in S$ we have $\sum_{i=1}^{n} w_{i}^{\bar{s}} x_{i} \leq \sum_{i=1}^{n} w_{i}^{s} x_{i}$; by taking the minimum over $s$, we get $\sum_{i=1}^{n} w_{i}^{\bar{s}} x_{i} \leq \min _{s \in S} \sum_{i=1}^{n} w_{i}^{s} x_{i}$. By further taking the maximum over $X$, we obtain $\max _{X \in K} \sum_{i=1}^{n} w_{i}^{\bar{s}} x_{i} \leq \max _{X \in K} \min _{s \in S} \sum_{i=1}^{n} w_{i}^{s} x_{i}$; that is $\Delta_{\bar{s}} \leq \Delta$. We conclude that $\Delta=\Delta_{\bar{s}}$.

Corollary 3.1 The problem AbRMWIS can be solved in polynomial time when the set $S$ of scenarios is a Cartesian product.

Proof This result follows from Proposition 3.4 and the fact that $\mathrm{MWIS}_{\bar{s}}$ can be solved in polynomial time $[5,11,12,14]$.

\section{Min-max regret}

This section is devoted to the minimization of the maximum regret (min-max regret), defined as:

$$
\text { (ReRMWIS) } \quad \min _{X \in K} \max _{s \in S} F_{s}^{*}-F_{s}(X)
$$

A result similar to that of Proposition 3.1 holds.

Proposition 4.1 ReRMWIS is NP-hard even when $S$ contains only two scenarios. 
Proof Consider the reduction used for the proof of Proposition 3.1. Observe that the total weight of the maximum weighted independent set for each scenario in isolation is the same, i.e. $\delta=F_{1}^{*}=F_{2}^{*}=\sum_{i=1}^{n} \max \left\{a_{2 i-1}, a_{2 i}\right\}$. The same reasoning as in the proof of Proposition 3.1 leads to the conclusion that the optimal value of ReRMWIS is less than or equal to $\theta=\delta-Q$ if and only if the EOPP instance is a YES instance.

Proposition 4.2 ReRMWIS can be solved in pseudo-polynomial time when $S$ is bounded.

Algorithm 1 can be used to solve ReRMWIS in pseudo-polynomial time if the following modifications are made:

1. In line 5, compute $F_{n+1}\left(\alpha_{1}, \ldots, \alpha_{|S|}\right)=\max _{s \in S}\left\{F_{s}^{*}-\alpha_{s}\right\}$.

2. In line 12 , use min instead of max.

The time complexity of the algorithm remains the same.

Proposition 4.3 ReRMWIS is strongly NP-hard when $S$ is unbounded.

Proof Consider exactly the same layered graph $G$ as built in the proof of Proposition 3.3. Notice that the optimal objective values for each scenario $s \in S$ individually are $F_{s}^{*}=m B$. The same reasoning as in the proof of Proposition 3.3 leads to the conclusion that there exists a 3-partition if and only if ReRMWIS has an optimal value less than or equal to $(m-1) B$.

Next, we again consider a Cartesian scenario set. Recall that in this case $S=\prod_{i=1}^{n} W_{i}$, where the set $W_{i}$ contains all the possible weights of vertex $i$. We define $L_{i}=\min \left\{w \in W_{i}\right\}$ and $U_{i}=\max \left\{w \in W_{i}\right\}$ and let $W_{i}^{\prime}=\left\{L_{i}, U_{i}\right\}$ ( $W_{i}^{\prime}$ is a singleton if $L_{i}=U_{i}$ ). We define a new scenario set with at most $2^{n}$ scenarios as $S^{\prime}=\prod_{i=1}^{n} W_{i}^{\prime}$.

Proposition 4.4 When $S$ is a Cartesian product, a solution to ReRMWIS can be obtained by solving a reduced instance where $S$ is replaced by $S^{\prime}$.

Proof Let $X \in K$. We define $Z(X)=\max \left\{F_{s}^{*}-F_{s}(X): s \in S\right\}$ and $Z^{\prime}(X)=$ $\max \left\{F_{s}^{*}-F_{s}(X): s \in S^{\prime}\right\}$ and we want to show that $Z(X)=Z^{\prime}(X)$. Clearly, $Z(X) \geq Z^{\prime}(X)$ since $S^{\prime} \subseteq S$. Let $s_{0} \in S$ such that $Z(X)=F_{s_{0}}^{*}-F_{s_{0}}(X)$. If $s_{0} \in S^{\prime}$ then $Z(X)=Z^{\prime}(X)$; otherwise $\left(s_{0} \notin S^{\prime}\right)$ let $X^{0} \in K$ such that $F_{s_{0}}^{*}=\sum_{i=1}^{n} w_{i}^{s_{0}} x_{i}^{0}$; then consider the scenario $s_{1} \in S^{\prime}$ defined as follows. If $x_{i}^{0}=1$ then $w_{i}^{s_{1}}=U_{i}$, else $w_{i}^{s_{1}}=L_{i}$. We have $F_{s_{0}}^{*} \leq \sum_{i=1}^{n} w_{i}^{s_{1}} x_{i}^{0} \leq F_{s_{1}}^{*}$. Moreover,

$$
\begin{aligned}
Z^{\prime}(X) \geq F_{s_{1}}^{*}-\sum_{i=1}^{n} w_{i}^{s_{1}} x_{i} & \geq \sum_{i=1}^{n} w_{i}^{s_{1}} x_{i}^{0}-\sum_{i=1}^{n} w_{i}^{s_{1}} x_{i} \\
& \geq \sum_{i=1}^{n} w_{i}^{s_{1}}\left(x_{i}^{0}-x_{i}\right) \geq \sum_{i=1}^{n} w_{i}^{s_{0}}\left(x_{i}^{0}-x_{i}\right)=Z(X) .
\end{aligned}
$$

Therefore, $Z(X)=Z^{\prime}(X)$ and by taking the minimum over $X \in K$ we have the required proof.

Notice that Proposition 4.4 does not imply straightforward complexity results because the scenario set is still a Cartesian product. 


\section{Conclusions}

In this paper, we have studied the robust maximum weighted independent-set problem on interval graphs with two different criteria, namely the absolute robustness criterion and the min-max regret criterion. For a discrete scenario set, we prove that the problem is NP-hard for each of the robustness criteria but solvable in pseudo-polynomial time when there is a constant number of scenarios. We show that the problem is strongly NP-hard when the set of scenarios is unbounded. For the scenario set given as a Cartesian product, the problem is equivalent to a maximum weighted independent-set problem on the same interval graph but without uncertainty for the first objective function, and we find that the scenario set can be reduced for the second objective function.

\section{Acknowledgements}

The authors thank Frits Spieksma (KU Leuven) and Reviewer 1 for useful comments on an earlier version of this paper.

\section{References}

1. I. Averbakh. On the complexity of a class of combinatorial optimization problems with uncertainty. Mathematical Programming, Series A, 90:263-272, 2001.

2. S. Basagni. Finding a maximal weighted independent set in wireless networks. Telecommunication Systems, 18:155-168, 2001.

3. D. Bertsimas and M. Sim. Robust discrete optimization and network flows. Mathematical Programming, Series B, 98:49-71, 2003.

4. D. Bertsimas and M. Sim. The price of robustness. Operations Research, 52:35-53, 2004.

5. A. Frank. Some polynomial algorithms for certain graphs and hypergraphs. In 5 th British Combinatorial Conference, pages 211-226, 1975.

6. F. Gardi. Mutual exclusion scheduling with interval graphs or related classed: complexity and algorithms. 4OR, 1:88-90, 2006.

7. M.R. Garey and D.S. Johnson. Computers and Intractability: A Guide to the Theory of NP-Completeness. W.H. Freeman and Co., 1979.

8. F. Gavril. Maximum weight independent sets and cliques in intersection graphs of filaments. Information Processing Letters, 73:181-188, 2000.

9. M.C. Golumbic. Algorithmic Graph Theory and Perfect Graphs. Elsevier B.V., 2004.

10. P. Kouvelis and G. Yu. Robust Discrete Optimization and its Applications. Kluwer Academic Publishers, Norwell, MA, 1997.

11. S. Mandal and M. Pal. Maximum weight independent set of circular-arc graph and its application. Journal of Applied Mathematics and Computing, 22:161-174, 2006.

12. M. Pal and G.P. Bhattacharjee. A sequential algorithm for finding a maximum weight $k$-independent set on interval graphs. International Journal of Computer Mathematics, 60:205-214, 1996.

13. A.P. Ruszczyński and A. Shapiro. Stochastic Programming. Elsevier, 2003.

14. A. Saha, M. Pal, and T.K. Pal. Selection of programme slots of television channels for giving advertisement: A graph theoretic approach. Information Sciences, 177:2480$2492,2007$. 